\title{
Participant Contact List
}

Abboud Werner, Sherry

University of Texas Health Science Center, San Antonio, Tex., USA

abboudwerner@uthscsa.edu

Alvares, Keith

Northwestern University, Chicago, III., USA

k-alvares@northwestern.edu

Anderson, Paul

Queen Mary University, London, UK

p.anderson@qmul.ac.uk

Ascenzi, Maria-Grazia

University of California at Los Angeles, Los Angeles, Calif., USA

mgascenzi@mednet.ucla.edu

Babajko, Sylvie

University of Paris, Paris, France

sylvie.babajko@crc.jussieu.fr

Bahar, Hila

Harvard University, Boston, Mass., USA

bahar.hila@mgh.harvard.edu

Bartlett, John D.

Forsyth Institute, Boston, Mass., USA

jbartlett@forsyth.org

Beniash, Elia

University of Pittsburgh, Pittsburgh, Pa., USA

ebeniash@pitt.edu

Bentov, Shmuel

Ben Gurion University, Beersheva, Israel

bentovs@bgu.ac.il

Berdal, Ariane

University of Paris, Paris, France

ariane.berdal@crc.jussieu.fr
Blank, Robert D.

University of Wisconsin, Madison, Wisc., USA

rdb@medicine.wisc.edu

Bonewald, Lynda F.

University of Missouri, Kansas City, Mo., USA

bonewaldl@umkc.edu

Boonrungsiman, Suwimon

Imperial College, London, UK

s.boonrungsiman07@imperial.ac.uk

Boskey, Adele L.

Hospital for Special Surgery, New York, N.Y., USA

boskeya@hss.edu

Boyan, Barbara D.

Georgia Polytechnical Institute, Atlanta, Ga., USA

barbara.boyan@bme.gatech.edu

Bringas, Pablo

University of Southern California, Los Angeles, Calif., USA

bringas@usc.edu

Bromage, Timothy G.

New York University, New York City, N.Y., USA

tim.bromage@nyu.edu

Bromley, Keith M.

University of Southern California, Los Angeles, Calif., USA

kbromley@usc.edu

Buchko, Garry W.

Pacific Northwest National Laboratory, Richland, Wash., USA

garry.buchko@pnl.gov

Budell, Chase

City University of New York at Brooklyn, New York, N.Y., USA wcbudell@gmail.com

\section{KARGER \\ Fax +4161306 1234 \\ E-Mail karger@karger.ch}

www.karger.com (c) 2011 S. Karger AG, Basel

1422-6405/11/1944-0340\$38.00/0

Accessible online at: www.karger.com/cto 
Chan, Bin San (Vera)

University of Hong Kong, Pokfulam, China

verabschan@yahoo.com

Chaudhri, Reyhaan A.

Georgia Polytechnical Institute, Atlanta, Ga., USA

reyhaan.chaudhri@bme.gatech.edu

Chaussain, Catherine

University of Paris Descartes, Paris, France

catherine.chaussain@parisdescartes.fr

Chen, Shuo

University of Texas Health Science Center, San Antonio, Tex., USA

chens0@uthscsa.edu

Chen, Chun-Long

Lawrence Berkeley National Laboratory, Berkeley, Calif., USA

chunlongchen@lbl.gov

Chen, Haiyan

University of Alabama at Birmingham, Birmingham, Ala., USA

hychen@uab.edu

Chun, Yong-Hee Patricia

University of Texas Health Science Center, San Antonio, Tex., USA

chuny@uthscsa.edu

De Yoreo, Jim

Lawrence Berkeley National Laboratory, Berkeley, Calif., USA

jjdeyoreo@lbl.gov

Dechow, Paul

Texas A\&M Health Science Center, Dallas, Tex., USA

pdechow@bcd.tamhsc.edu

DeLucas, Lawrence

University of Alabama at Birmingham, Birmingham, Ala., USA

delucas@cbse.uab.edu

DenBesten, Pamela

University of California, San Francisco, Calif., USA

pamela.denbesten@ucsf.edu

Deshpande, Atul Suresh

University of Pittsburgh, Pittsburgh, Pa., USA

atd12@pitt.edu

Diekwisch, Thomas

University of Illinois, Chicago, III., USA

tomdkw@uic.edu

Donahue, Henry J.

Pennsylvania State University, Hershey, Pa., USA

hdonahue@hmc.psu.edu
Donnelly, Eve

Hospital for Special Surgery, New York, N.Y., USA

donnellye@hss.edu

Dorvee, Jason $\mathrm{R}$.

Cornell University, Ithaca, N.Y., USA

jrd44@cornell.edu

Dowd, Terry L.

City University of New York at Brooklyn, New York, N.Y., USA

TDowd@brooklyn.cuny.edu

Drummond, James L.

National Institute for Dental and Craniofacial Research,

Bethesda, Md., USA

drummondj@nidcr.nih.gov

D'Souza, Rena N.

Texas A\&M Health Science Center, Dallas, Tex., USA

rdsouza@bcd.tamhsc.edu

Erlandsen, Heidi

University of Alabama at Birmingham, Birmingham, Ala., USA

herlands@uab.edu

Evans, John S.

New York University, New York City, N.Y., USA

jse1@nyu.edu

Everett, Eric T.

University of North Carolina, Chapel Hill, N.C., USA

eric_everett@dentistry.unc.edu

Fan, Daming

University of Southern California, Los Angeles, Calif., USA

daming@usc.edu

Fang, Ping-An

University of Pittsburgh, Pittsburgh, Pa., USA

paf30@pitt.edu

Feng, Jian

Texas A\&M Health Science Center, Dallas, Tex., USA

jfeng@bcd.tamhsc.edu

Fisher, Larry W.

National Institute for Dental and Craniofacial Research,

Bethesda, Md., USA

Ifisher@dir.nidcr.nih.gov

Fratzl, Peter

Max Planck Institute, Potsdam, Germany

Fratzl@mpikg.mpg.de

Galler, Kerstin

University of Regensburg, Regensburg, Germany

kerstin.galler@klinik.uni-regensburg.de 
George, Anne

University of Illinois, Chicago, III., USA

anneg@uic.edu

Gibson, Carolyn W.

University of Pennsylvania, Philadelphia, Pa., USA

cgibson@dental.upenn.edu

Gittens, Rolando A.

Georgia Polytechnical Institute, Atlanta, Ga., USA

rgittensi@gatech.edu

Goldberg, Michel

University of Paris Descartes, Paris, France

michel.goldberg@parisdescartes.fr

Goldberg, Harvey A.

University of Western Ontario, London, Ont., Canada

hagoldbe@uwo.ca

Gordon, Jonathan A.R.

University of Massachusetts, Worcester, Mass., USA

jonathan.gordon@umassmed.edu

Gorski, Jeffrey P.

University of Missouri, Kansas City, Mo., USA

Gorskij@umkc.edu

Gower, Laurie B.

University of Florida, Gainesville, Fla., USA

lgowe@mse.ufl.edu

Groppe, Jay C.

Texas A\&M Health Science Center, Dallas, Tex., USA

jgroppe@bcd.tamhsc.edu

Grynpas, Marc

University of Toronto, Toronto, Ont., Canada

grynpas@mshri.on.ca

Gullard, Angela

University of Alabama at Birmingham, Birmingham, Ala., USA

agullard@uab.edu

Han, Xianglong

Texas A\&M Health Science Center, Dallas, Tex., USA

xianglonghan@163.com

Holcroft, James

University of Toronto, Toronto, Ont., Canada

james.holcroft@utoronto.ca

Hotton, Dominic

University of Paris, Paris, France

dominique.hotton@crc.jussieu.fr

Hug, Susanna

University of Western Ontario, London, Ont., Canada

shug@uwo.ca
Hunter, Graeme K.

University of Western Ontario, London, Ont., Canada

graeme.hunter@schulich.uwo.ca

Huser, Thomas

University of California at Davis, Sacramento, Calif., USA

trhuser@ucdavis.edu

Jacques, Jaime

University of Paris, Paris, France

jaimejacques@gmail.com

Jacquet, Robin

University of Akron, Akron, Ohio, USA

rmj@uakron.edu

Javed, Amjad

University of Alabama at Birmingham, Birmingham, Ala., USA

javeda@uab.edu

Joester, Derk

Northwestern University, Evanston, III., USA

d-joester@northwestern.edu

Kawasaki, Kazuhiko

Pennsylvania State University, University Park, Pa., USA

kuk2@psu.edu

Krebsbach, Paul H.

University of Michigan, Ann Arbor, Mich., USA

paulk@umich.edu

Kuijpers, Gemma

Food and Drug Administration, Silver Spring, Md., USA

gemma.kuijpers@fda.hhs.gov

Lacruz, Rodrigo S.

University of Southern California, Los Angeles, Calif., USA

rodrigo@usc.edu

Landis, William J.

University of Akron, Akron, Ohio, USA

wlandis@uakron.edu

Lange, Claudia

Max Planck Institute, Potsdam, Germany

Claudia.Lange@mpikg.mpg.de

Lee, Christopher S.D.

Emory University and Georgia Tech, Atlanta, Ga., USA

cslee7@emory.edu

Li, Bei

University of Southern California, Los Angeles, Calif., USA

bl_005@usc.edu

Lian, Jane

University of Massachusetts, Worcester, Mass., USA

jane.lian@umassmed.edu 
Lim, Mei Ling

Karolinska Institute, Stockholm, Sweden

Meiling.Lim@gmail.com

Liu, Yi

University of Southern California, Los Angeles, Calif., USA

liu14@usc.edu

Liu, Xiaohua

Texas A\&M Health Science Center, Dallas, Tex., USA

xliu@bcd.tamhsc.edu

Lu, Yongbo (Bob)

Texas A\&M Health Science Center, Dallas, Tex., USA

ylu@bcd.tamhsc.edu

MacDougall, Mary

University of Alabama at Birmingham, Birmingham, Ala., USA

macdougall@uab.edu

Margolis, Henry C.

Forsyth Institute, Boston, Mass., USA

hmargolis@forsyth.org

Martinez-Avila, Olga

University of California at San Francisco, Berkeley, Calif., USA

olga.martinez@ucsf.edu

Masic, Admir

Max Planck Institute, Potsdam, Germany

Admir.Masic@mpikg.mpg.de

McDaniel, Jennifer S.

University of Texas Health Science Center, San Antonio, Tex., USA

s.jennifer@sbcglobal.net

McDougall, Carmel

University of Queensland, Brisbane, Qld., Australia

c.mcdougall@uq.edu.au

McKee, Marc D.

McGill University, Montreal, Que., Canada

marc.mckee@mcgill.ca

Midura, Ronald J.

The Cleveland Clinic, Cleveland, Ohio, USA

midurar@ccf.org

Millán, José Luis

Sanford-Burnham Medical Research Institute, La Jolla, Calif., USA

millan@sanfordBurnham.org

Mina, Mina

University of Connecticut Health Center, Farmington, Conn., USA

mina@nso1.uchc.edu
Moradian-Oldak, Janet

University of Southern California, Los Angeles, Calif., USA

joldak@usc.edu

Murdock, Duncan

University of Bristol, Bristol, UK

Duncan.Murdock@bris.ac.uk

Murshed, Monzur

McGill University, Montreal, Que., Canada

monzur.murshed@mcgill.ca

Nakayama, Yohei

University of Toronto, Toronto, Ont., Canada

yohei.nakayama@utoronto.ca

Nikdin, Hero

Karolinska Institute, Stockholm, Sweden

hero.nikdin@ki.se

Ogata, Yorimasa

Nihon University, Chiba, Japan

ogata.yorimasa@nihon-u.ac.jp

Omelon, Sidney

University of Ottawa, Ottawa, Ont., Canada

somelon@uottawa.ca

Paine, Michael L.

University of Southern California, Los Angeles, Calif., USA

paine@usc.edu

Papagerakis, Petros

University of Michigan, Ann Arbor, Mich., USA

petrosp@umich.edu

Prasad, Monica

Texas A\&M Health Science Center, Dallas, Tex., USA

Mprasad@bcd.tamhsc.edu

Pugach, Megan K.

University of Pennsylvania, Philadelphia, Pa., USA

mpugach@biochem.dental.upenn.edu

Qin, Haiyan

Texas A\&M Health Science Center, Los Angeles, Calif., USA

hqin@usc.edu

Qin, Chunlin

Texas A\&M Health Science Center, Dallas, Tex., USA

cqin@bcd.tamhsc.edu

Qu, Cunye

University of Southern California, Los Angeles, Calif., USA cqu@usc.edu

Rangiani, Afsaneh

Texas A\&M Health Science Center, Dallas, Tex., USA

arangiani@bcd.tamhsc.edu 
Ravindran, Sriram

University of Illinois, Chicago, III., USA

sravin1@uic.edu

Reeve, Jonathan

Cambridge University, Cambridge, UK

jr357@cam.ac.uk

Regan, Katherine R.

Texas A\&M Health Science Center, Dallas, Tex., USA

kr2@rice.edu; kregan@bcd.tamhsc.edu

Ren, Changchun

University of Alabama at Birmingham, Birmingham, Ala., USA

cren@uab.edu

Rez, Peter

Arizona State University, Tempe, Ariz., USA

peter.rez@asu.edu

Sagomonyants, Karen

University of Connecticut Health Center, Farmington, Conn., USA

Ksagomonyants@student.uchc.edu

Salmon, Benjamin

University of Paris, Paris, France

drbenjaminsalmon@hotmail.fr

Scheller, Erica L.

University of Michigan, Ann Arbor, Mich., USA

scheller@umich.edu

Serra, Rosa

University of Alabama at Birmingham, Birmingham, Ala., USA

rserra@uab.edu

Seto, Jong

Max Planck Institute, Potsdam, Germany

jong.seto@mpikg.mpg.de

Sfeir, Charles

University of Pittsburgh, Pittsburgh, Pa., USA

csfeir@pitt.edu

Shapiro, Irving M.

Thomas Jefferson University, Philadelphia, Pa., USA

irving.shapiro@jefferson.edu

Shi, Songtao

University of Southern California, Los Angeles, Calif., USA

songtaos@usc.edu

Shore, Eileen

University of Pennsylvania, Philadelphia, Pa., USA

shore@mail.med.upenn.edu
Silverman, Lance D.

Yeshiva University, New York, N.Y., USA

Isilverm@yu.edu

Simmer, James $P$.

University of Michigan, Ann Arbor, Mich., USA

jsimmer@umich.edu

Sinha, Sourabh

Arizona State University, Tempe, Ariz., USA

sourabh.sinha@asu.edu

Sloan, Alastair

Cardiff University, Cardiff, UK

sloanaj@cardiff.ac.uk

Sone, Eli D.

University of Toronto, Toronto, Ont., Canada

eli.sone@utoronto.ca

Sricholpech, Marnisa

University of North Carolina, Chapel Hill, N.C., USA

sricholm@dentistry.unc.edu

Stevens, Molly

Queen Mary University, London, UK

m.stevens@imperial.ac.uk

Suder, Marzena

Jagiellonian University, Krakow, Poland

suderm@chemia.uj.edu.pl

Sun, Yao

Texas A\&M Health Science Center, Dallas, Tex., USA

ysun@bcd.tamhsc.edu

Syed-Picard, Fatima N.

University of Pittsburgh, Pittsburgh, Pa., USA

fns4@pitt.edu

Takai, Hideki

Nihon University, Chiba, Japan

takai.hideki@nihon-u.ac.jp

Tao, Jinhui

Lawrence Berkeley National Laboratory, Berkeley, Calif., USA

jtao@lbl.gov

Thula-Mata, Taili

University of Florida, Gainesville, Fla., USA

tthula@gmail.com

Veis, Arthur

Northwestern University, Chicago, III., USA

aveis@northwestern.edu

Vital, Sybille

University of Paris Descartes, Paris, France

sibylle.vital@parisdescartes.fr 
Voisey, Anika

Texas A\&M Health Science Center, Dallas, Tex., USA

avoisey@bcd.tamhsc.edu

von Marschall, Zofia

National Institute for Dental and Craniofacial Research,

Bethesda, Md., USA

vonmarschallz@mail.nih.gov

Waddington, Rachel

Cardiff University, Cardiff, UK

waddingtonrj@cardiff.ac.uk

Wallace, Joseph M.

University of Michigan, Ann Arbor, Mich., USA

jmwallac@umich.edu

Wang, Xiaofang

Texas A\&M Health Science Center, Dallas, Tex., USA

xwang@bcd.tamhsc.edu

Wang, Lei

University of Southern California, Los Angeles, Calif., USA wang65@usc.edu

Weiner, Steve

The Weizmann Institute, Rehovot, Israel

steve.weiner@weizmann.ac.il

Wright, J. Tim

University of North Carolina, Chapel Hill, N.C., USA

tim_wright@dentistry.unc.edu
Yamakoshi, Yasuo

University of Michigan, Ann Arbor, Mich., USA

yamasan@umich.edu

Yang, Yang

University of Wisconsin, Madison, Wisc., USA

yang@chem.wisc.edu

Yang, Xiudong

University of Southern California, Los Angeles, Calif., USA

xiudongy@usc.edu

Yoshizawa, Sayuri

University of Pittsburgh, Pittsburgh, Pa., USA

say15@pitt.edu

Zaslansky, Paul

Max Planck Institute, Potsdam, Germany

paul.zaslansky@mpikg.mpg.de

Zhang, Xueming

University of Alabama at Birmingham, Birmingham, Ala., USA

zhangxm@uab.edu

Zhang, Nianli

University of Wisconsin, Madison, Wisc., USA

nzhang5@wisc.edu

Zhu, Qinglin

Texas A\&M Health Science Center, Dallas, Tex., USA

zhu@bcd.tamhsc.edu 